\title{
Computational Methods for Identification of Vibrating Structures
}

\author{
F.J. Cara ${ }^{1}$, J. Carpio ${ }^{2}$, J. Juan ${ }^{2}$ and E. Alarcon ${ }^{1}$ \\ ${ }^{1}$ Department of Structural Mechanics and Industrial Constructions \\ ${ }^{2}$ Department of Organization Engineering, \\ Business Administration and Statistics \\ Polytechnical University of Madrid, Spain
}

\begin{abstract}
This paper describes a theoretical and experimental research work in relation to the system identification of an steel arch bridge. This bridge forms part of the Spanish highway network and is located about $30 \mathrm{~km}$ at the south of Granada (Spain). Ambient vibration tests were conducted during two days and the response of the structure was measured at selected points.

The scope of this paper is present some modal identification results obtained using state-of-the-art time domain system identification method (data-driven stochastic subspace algorithms) applied to the ambient vibration data measured in two of this points. Point parameter estimation and interval parameter estimation are presented. A complete method for interval parameter estimation using state space models and subspace system identification is developed. This method includes the construction of a plot similar to stabilization diagram which permits differentiate system modes from spurious modes for a given system order.
\end{abstract}

Additionally, the obtained experimental results are compared with those from a three dimensional finite element analysis.

Keywords: System identification in structures, State Space models, Kalman filter, Stochastic Subspace methods, Bootstrap

\section{Introduction}

"System identification deals with the problem of building mathematical models of dynamical systems based on observed data from the system" [1]. In the context of civil engineering, the system refers to a large scale structure such as a building, bridge, or an offshore structure, and identification mostly involves the determination of modal parameters (the natural frequencies, damping ratios, and mode shapes). 


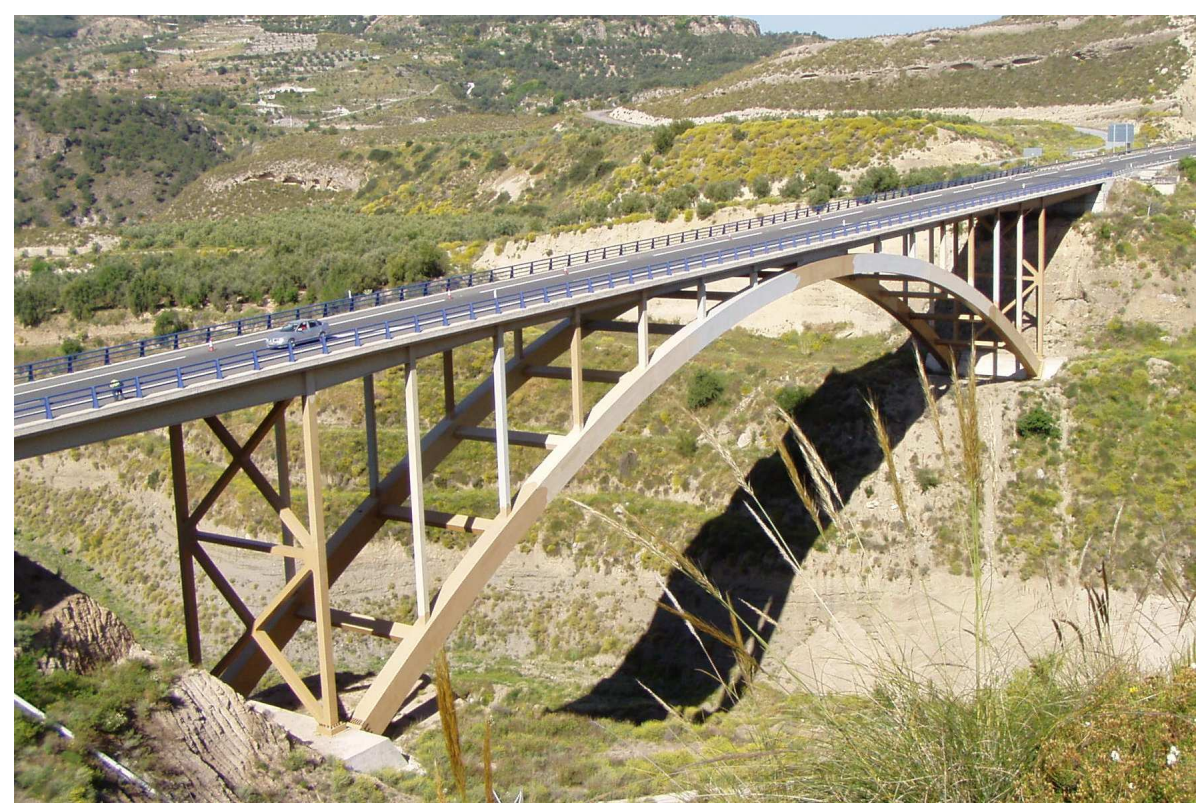

Figure 1: Tablate bridge picture

Parametric model based structural identification methods involve the use of mathematical models to represent structural system behavior in either time or frequency domain. The benefits of using parametric models for structural identification include their direct relationship with physically meaningful quantities such as stiffness and mass, improved accuracy and resolution, and their suitability for analysis, prediction, fault diagnosis and control.

Popular time domain parametric models used for structural identification purposes include: ARX models, ARMAX models, state space models, etc. Many identification algorithms are available to estimate the parameters of such parametric models, e.g. prediction error method (PEM), least squares estimation (LSE), maximum likelihood algorithm (MLA), eigensystem realization algorithm (ERA) and subspace method.

The scope of this paper is present some modal identification results obtained using state-of-the-art time domain system identification method (data-driven stochastic subspace algorithms) applied to the ambient vibration data measured in a steel arch bridge.

Point estimate and interval estimate have been carried out in state space model using these ambient vibration measurements. Stable modal parameters are found using stabilization diagram and bootstrap procedure is proposed for interval estimate of modal parameters.

Finally, the obtained experimental results are compared with those from a three dimensional finite element analysis. 


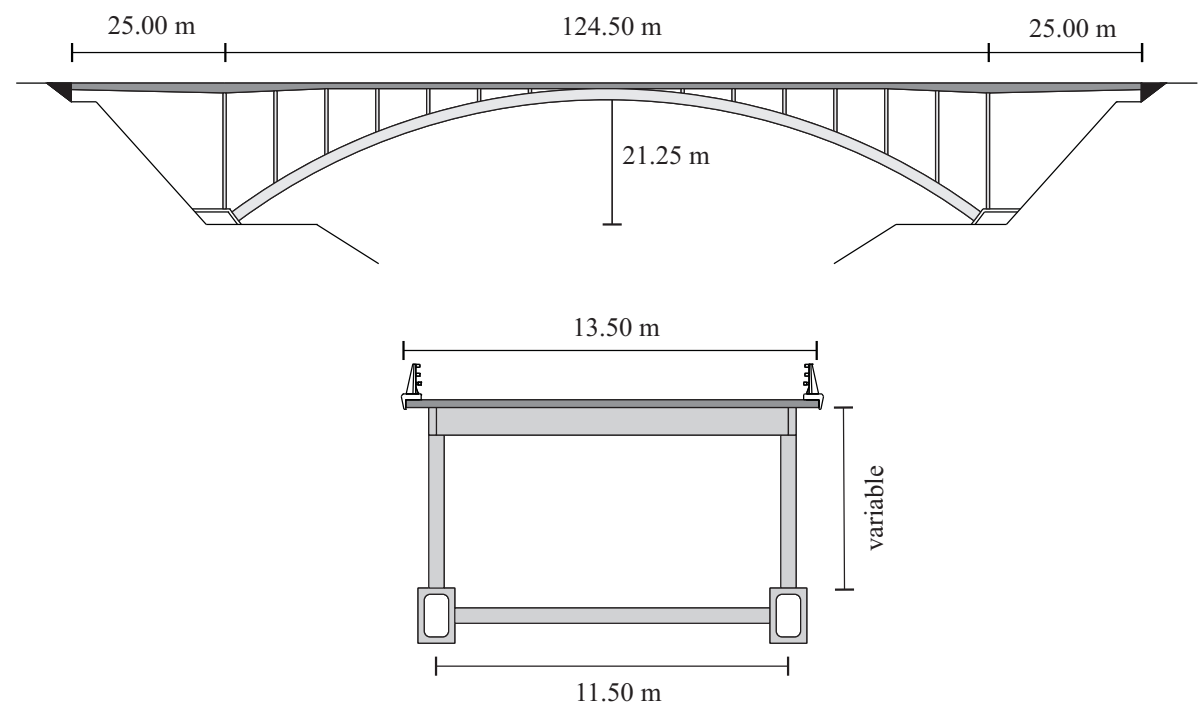

Figure 2: Elevation and deck cross-section of Tablate bridge

\section{Bridge description}

Tablate Bridge (Figure 1 and 2) forms part of the spanish highway network and is located about $30 \mathrm{~km}$ at the south of Granada (Spain). The total length of the bridge is $174.50 \mathrm{~m}$. The arch spans $124.50 \mathrm{~m}$ between abutments and rises $21.25 \mathrm{~m}$ until the crown. The ribs have a box cross section with external dimensions of $H / \mathrm{V}=1.20 / 1.80$ $\mathrm{m}$ and variable wall thickness throughout their length.

The superstructure is comprised of cast-inplace concrete deck (13.50 m wide) and transverse beams that are supported by two longitudinal double-T steel beams with variable height. In turn, these longitudinal beams are supported by the ribs by means of vertical box beams. The transverse beams act compositely with the deck.

\section{Finite element model}

A three dimensional finite element model (FEM) has been developed for the numerical analysis of the structure using as-built drawings of the bridge. Modal analysis was carried out using ANSYS.

The arch, supports, and the beams of the bridge were represented as two-node beam elements (BEAM4) with 6 degrees of freedom per node. The deck slab was modeled using four-node shell elements with 6 degrees of freedom per node (SHELL63). The bridge supports were modeled using spring elements (COMBIN14).

The full model consists of 622 BEAM4 elements, 252 SHELL63 elements and 36 COMBIN14 elements, resulting in 741 nodes. Figure 3 shows a full 3-D view of the finite element model of the bridge. 


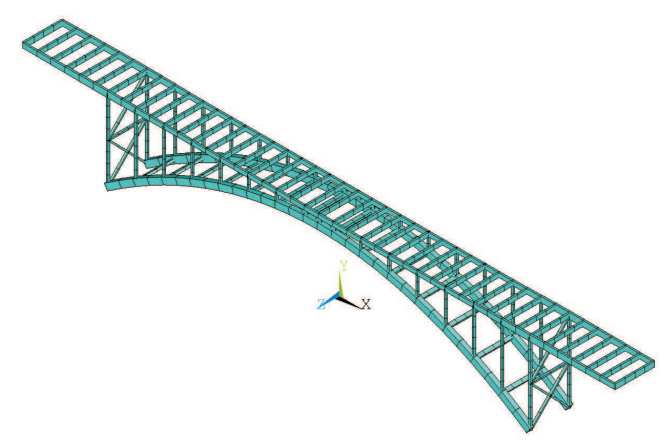

(a)

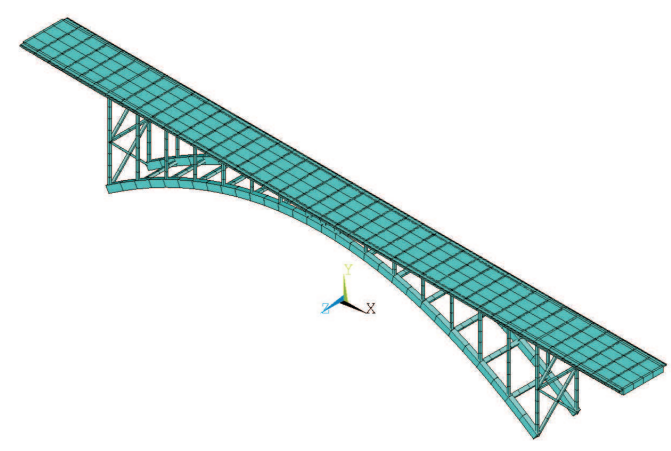

(b)

Figure 3: The 3-D FE model of Tablate bridge (a) steel strcuture (b) steel structure and concrete deck.

\section{Dynamic testing}

Ambient vibration tests were conducted during April 23-24, 2009. The response of the structure was measured at 26 selected points using Endevco accelerometers (model 7754-1000). Preliminary results obtained from a FE modal analysis were used to determine the optimum location of the sensors.

Eight accelerometers were available for the testing. Two stations of three sensors were formed (triaxial stations) and were placed at locations T1 and T2 (Figure 4). These sensors were held fixed for reference during the test. It is worth mentioning that in ambient vibration tests, where the input forces remain unknown and vary between the set-ups, different measurement setups can only be linked if there are some sensors in common. The reference stations ( $\mathrm{T} 1$ and $\mathrm{T} 2$ ) were chosen carefully in order to be able to measure the principal modes of interest of the bridge.

The two remaining sensors were placed at the different measurement points along the bridge deck, in which only vertical and transversal measurements were conducted (biaxial stations). In this work two set-ups will be analyzed, namely $(T 1, T 2, B 1)$ and $(T 1, T 2, B 2)$ respectively (Figure 4$)$.

Ambient vibration response were acquired during 900 seconds for all channels in both set-ups. The data were sampled to $4096 \mathrm{~Hz}$, and were decimated (order 7) to carry out data analysis in the frequency range of interest $(0-10 \mathrm{~Hz})$. All acceleration data were filtered using a Butterworth high-pass filter with cut-off frequency equal to $0.6 \mathrm{~Hz}$ to eliminate the mean. 


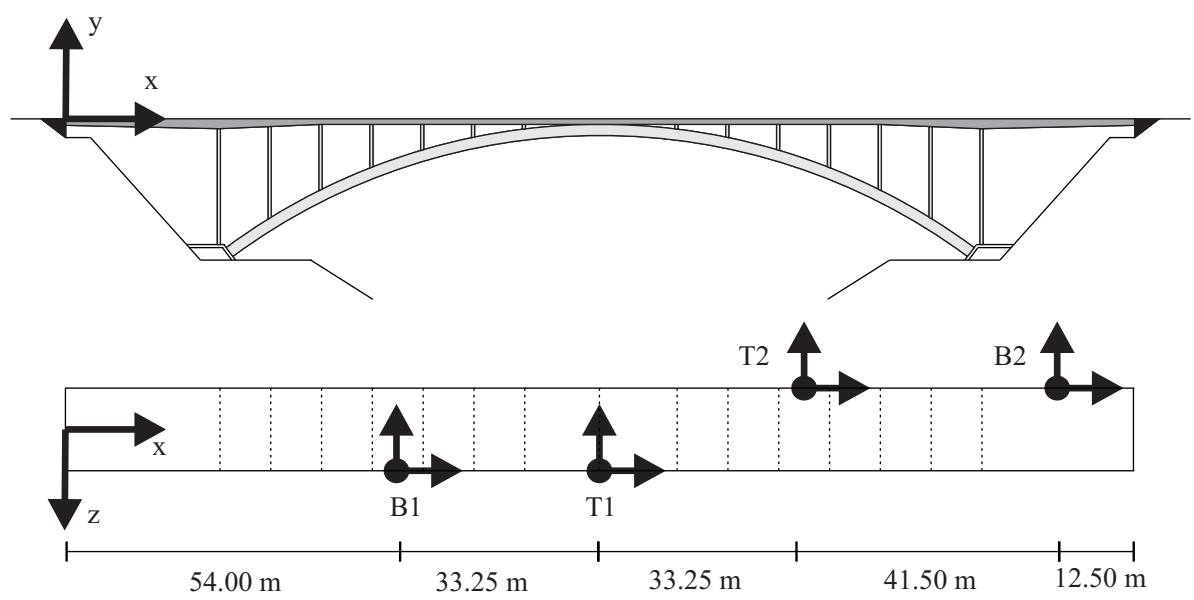

Figure 4: Layout of accelerometers along the bridge deck
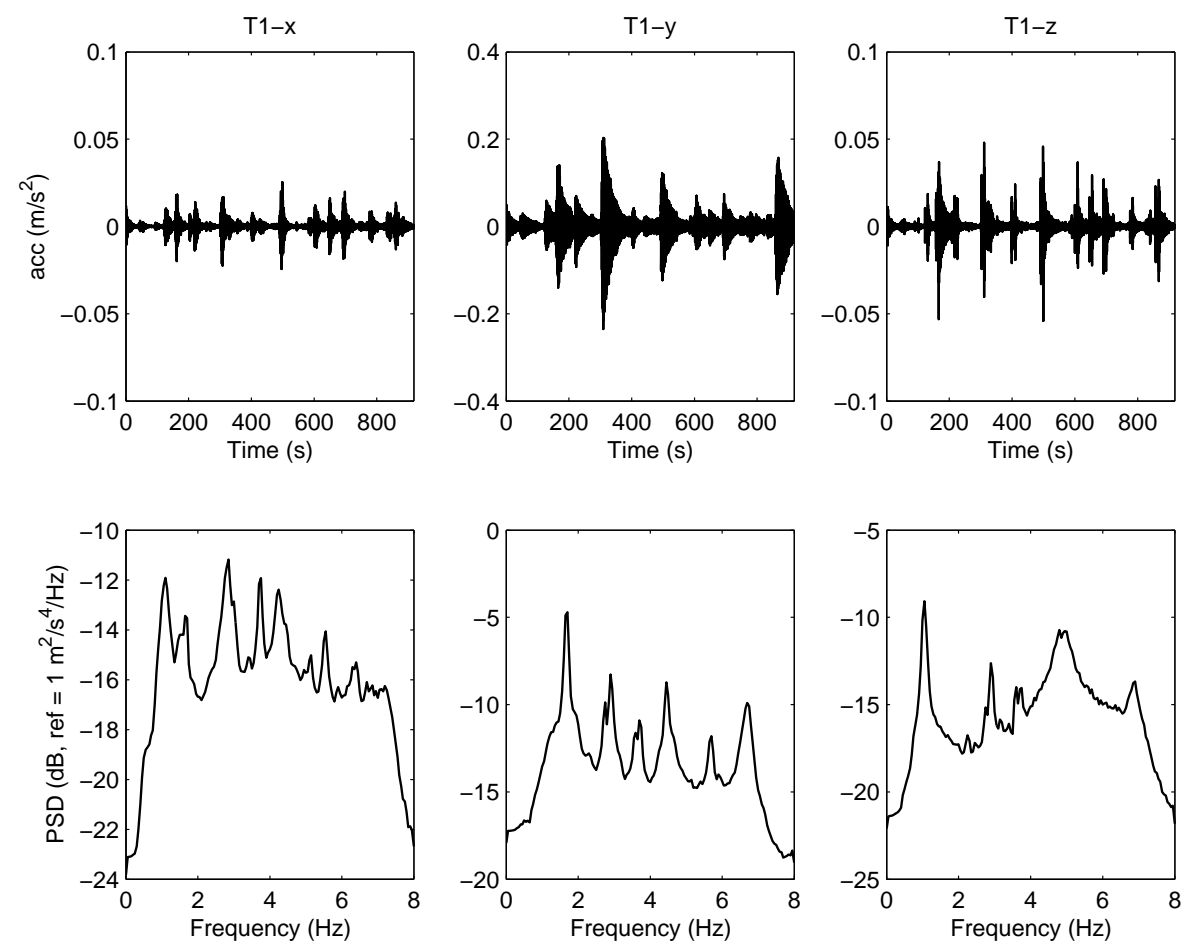

Figure 5: Bridge deck acceleration response measured in T1 for set-up (T1,T2,B2). 


\section{Modal identification using stochastic subspace me- thod}

In the field of civil engineering, system identification is applied mainly for modal analysis and Structural Health Monitoring. Modal analysis is based on the determination of modal parameters of a structural system which represent an optimal model, or basis, and can be used to describe the dynamics of a structural system. These modal parameters are modal frequencies, modal damping and modal vectors.

In the case of parametric system identification methods, the dynamic behavior of a system is described using mathematical models, and there are mathematical relationships between the modal parameters and the estimated model parameters. It is therefore common to use experimental modal analysis as a synonym for system identification. This section presents the identification of modal parameters in state space models.

\subsection{Point estimates of modal parameters}

\subsubsection{State space model}

It can be shown that a vibrating structure can be represented by a discrete-time stochastic state-space model given as:

$$
\begin{aligned}
& x_{k+1}=A x_{k}+B u_{k}+w_{k} \\
& y_{k}=C x_{k}+D u_{k}+v_{k}
\end{aligned}
$$

where

$k$ denotes the sampling instant $(t=k \Delta t$, with constant sampling time $\Delta t)$;

$y_{k} \in \mathbb{R}^{l}$ is the measured output vector;

$u_{k} \in \mathbb{R}^{m}$ is the measured input vector;

$x_{k} \in \mathbb{R}^{n}$ is the discrete state vector;

$w_{k} \in \mathbb{R}^{n}$ is the process noise due to disturbances and modeling inaccuracies;

$v_{k} \in \mathbb{R}^{l}$ is the measurement noise due to sensor inaccuracy

$A \in \mathbb{R}^{n \times n}$ is the transition state matrix describing the dynamics of the system (as characterised by its eigenvalues);

$B \in \mathbb{R}^{n \times m}$ is the input matrix;

$C \in \mathbb{R}^{l \times n}$ is the output matrix, which is describing how the internal state is transferred to the the output measurements $y_{k}$;

$D \in \mathbb{R}^{l \times m}$ is the direct transmission matrix; 
The noise vectors comprise unmeasurable vector signals assumed to be zero-mean with covariance matrices

$$
E\left[\left(\begin{array}{c}
w_{p} \\
v_{p}
\end{array}\right)\left(w_{p}^{T} v_{p}^{T}\right)\right]=\left[\begin{array}{cc}
Q & S \\
S^{T} & R
\end{array}\right] \delta_{p q}
$$

where $E$ is the expected value operator and $\delta_{p q}$ is the Kronecker delta.

In the practical problem of civil engineering structures, the reality is that only the responses of a structure $y_{k}$ are measured, while the input sequence $u_{k}$ remains unmeasured. In the case of ambient vibration testing, it is impossible to distinguish the input term $u_{k}$ from the noise terms $w_{k}, v_{k}$ in (1). Modeling the input term $u_{k}$ by the noise terms $w_{k}, v_{k}$, results in a purely stochastic system:

$$
\begin{aligned}
x_{k+1} & =A x_{k}+w_{k} \\
y_{k} & =C x_{k}+v_{k}
\end{aligned}
$$

The input is now implicitly modeled by the noise terms $w_{k}, v_{k}$. However the white noise assumptions of these noise terms cannot be omitted and (2) remain still applicable in equation (3).

\subsubsection{Stochastic subspace identification method for state space models}

The system identification problem in the state space model defined in Equation (3) can be formulated as the determination of the order $n$ and the corresponding system matrices $\mathrm{A}$ and $\mathrm{C}$ (up to within a similarity transformation) using the output measurements $\left\{y_{1}, y_{2}, \ldots, y_{l}\right\}$ available for $l$ time steps. There are several techniques to realize system identification, but we use in this work data-driven stochastic subspace identification method (SSI-DATA).

Let us for a moment assume that not only is $y_{k}$ measured, but also the sequence of state vectors $x_{k}$. Thus, with known $y_{k}$ and $x_{k}$, the model (3) becomes a linear regression. To see this clearly, let:

$$
\begin{array}{cr}
Z_{k}=\left[\begin{array}{c}
x_{k+1} \\
y_{k}
\end{array}\right] \in \mathbb{R}^{(n+l) \times 1} & \Phi_{k}=x_{k} \in \mathbb{R}^{n \times 1} \\
\theta=\left[\begin{array}{c}
A \\
C
\end{array}\right] \in \mathbb{R}^{(n+l) \times n} & E_{k}=\left[\begin{array}{c}
w_{k} \\
v_{k}
\end{array}\right] \in \mathbb{R}^{(n+l) \times 1}
\end{array}
$$

Then, (3) can be rewritten as:

$$
Z_{k}=\theta \Phi_{k}+E_{k}
$$

From this, all the matrix elements in $\theta$ can be estimated by the simplest least squares method as follows. The criterion function is defined as: 


$$
V_{N}(\theta)=\frac{1}{N} \sum_{k=1}^{N}\left(Z_{k}-\theta \Phi_{k}\right)^{2}
$$

The least square estimate $\hat{\theta}$ is defined by minimization of $V_{N}(\theta)$. Analytically, setting the gradient of $V_{N}(\theta)$ with respect to $\theta$ to zero, yields:

$$
\hat{\theta}=\left(\sum_{k=1}^{N} Z_{k} \Phi_{k}^{T}\right)\left(\sum_{k=1}^{N} \Phi_{k} \Phi_{k}^{T}\right)^{-1}
$$

Moreover, the the residuals and its covariance matrices are given by:

$$
\begin{gathered}
\hat{E}_{k}=Z_{k}-\hat{\theta} \Phi_{k} \\
{\left[\begin{array}{cc}
\hat{Q} & \hat{S} \\
\hat{S}^{T} & \hat{R}
\end{array}\right]=\frac{1}{N} \sum_{k=1}^{N} \hat{E}_{k} \hat{E}_{k}^{T}}
\end{gathered}
$$

Thus, knowing a sequence of state vectors $x_{k}$, the problem given by (4) is solved and the unknown matrices $\mathrm{A}$ and $\mathrm{C}$ are computed. Subspace methods are, in essence, numerical methods to construct a good estimate of a sequence of state vectors of the state space model from the measured output data.

In the following it is briefly explained how subspace methods work. First, the stochastic system (3) can be converted into a so-called forward innovation model by applying a Kalman filter:

$$
\begin{aligned}
x_{k+1} & =A x_{k}+K e_{k} \\
y_{k} & =C x_{k}+e_{k}
\end{aligned}
$$

Then, a non-steady state Kalman filter state estimate $\hat{x}_{k}$ is defined by the following recursive formulae:

$$
\hat{x}_{k}=A \hat{x}_{k-1}+K\left(y_{k-1}-C \hat{x}_{k-1}\right)
$$

the Kalman filter state estimate can be written as [2]:

$$
\hat{x}_{k}=L_{k}\left[\begin{array}{c}
y_{0} \\
y_{1} \\
\ldots \\
y_{k-1}
\end{array}\right]
$$

A linear combination of the past output measurements $y_{0}, \ldots, y_{k-1}\left(L_{k} \in \mathbb{R}^{n \times(k l)}\right)$, which allows for the definition of the Kalman filter state sequence of $j$ states as: 


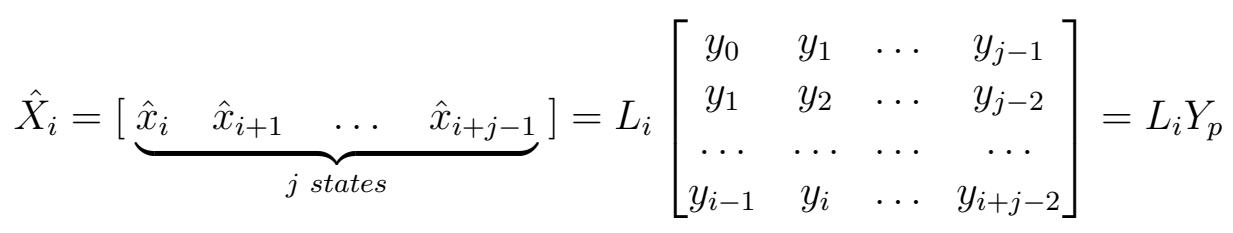

where $Y_{p}$ is the block Hankel matrix of past outputs.

$$
Y_{p}=\left[\begin{array}{cccc}
y_{0} & y_{1} & \ldots & y_{j-1} \\
y_{1} & y_{2} & \cdots & y_{j-2} \\
\cdots & \cdots & \cdots & \cdots \\
y_{i-1} & y_{i} & \ldots & y_{i+j-2}
\end{array}\right]
$$

In other words, $Y_{p}$ forms a row basis for the computation of the state sequence needed in (4). Nevertheless, subspace methods don't compute $\hat{X}_{i}$ directly from $Y_{p}$, but from a projection onto $Y_{p}$.

$$
\hat{X}_{i}=\Gamma_{i}^{-1}\left[Y_{f} / Y_{p}\right]
$$

where:

- $\Gamma_{i}$ is the extended observability matrix.

- $Y_{f}$ is the block Hankel matrix of future outputs, defined in a similar way like $Y_{p}$, but starting from $i$.

$$
Y_{f}=\left[\begin{array}{cccc}
y_{i} & y_{i+1} & \ldots & y_{i+j-1} \\
y_{i+1} & y_{i+2} & \ldots & y_{i+j} \\
\ldots & \cdots & \cdots & \cdots \\
y_{2 i-1} & y_{2 i} & \cdots & y_{2 i+j-2}
\end{array}\right]
$$

- $\left[Y_{f} / Y_{p}\right]$ is the orthogonal projection of $Y_{f}$ onto $Y_{p}$. This projection is computed using LQ decomposition.

Suppose that the singular value decomposition of $\left[Y_{f} / Y_{p}\right]$ be given by $\left[Y_{f} / Y_{p}\right]=$ $U S V^{T}$ with $\operatorname{rank}(S)=n$. Thus, the extended observability matrix can be taken as $\Gamma_{i}=U S^{1 / 2}$. Hence, it follows that the state sequence estimate is given by

$$
\hat{X}_{i}=S^{1 / 2} V^{T}
$$

This is the subspace projection approach, that applied robust numerical techniques like LQ decomposition and singular value decomposition to Hankel matrices formed with outputs measurements only to estimating the matrices of the state space model (a detailed description of different algorithms which implement subspace identification can be found in [2] and [9]). 

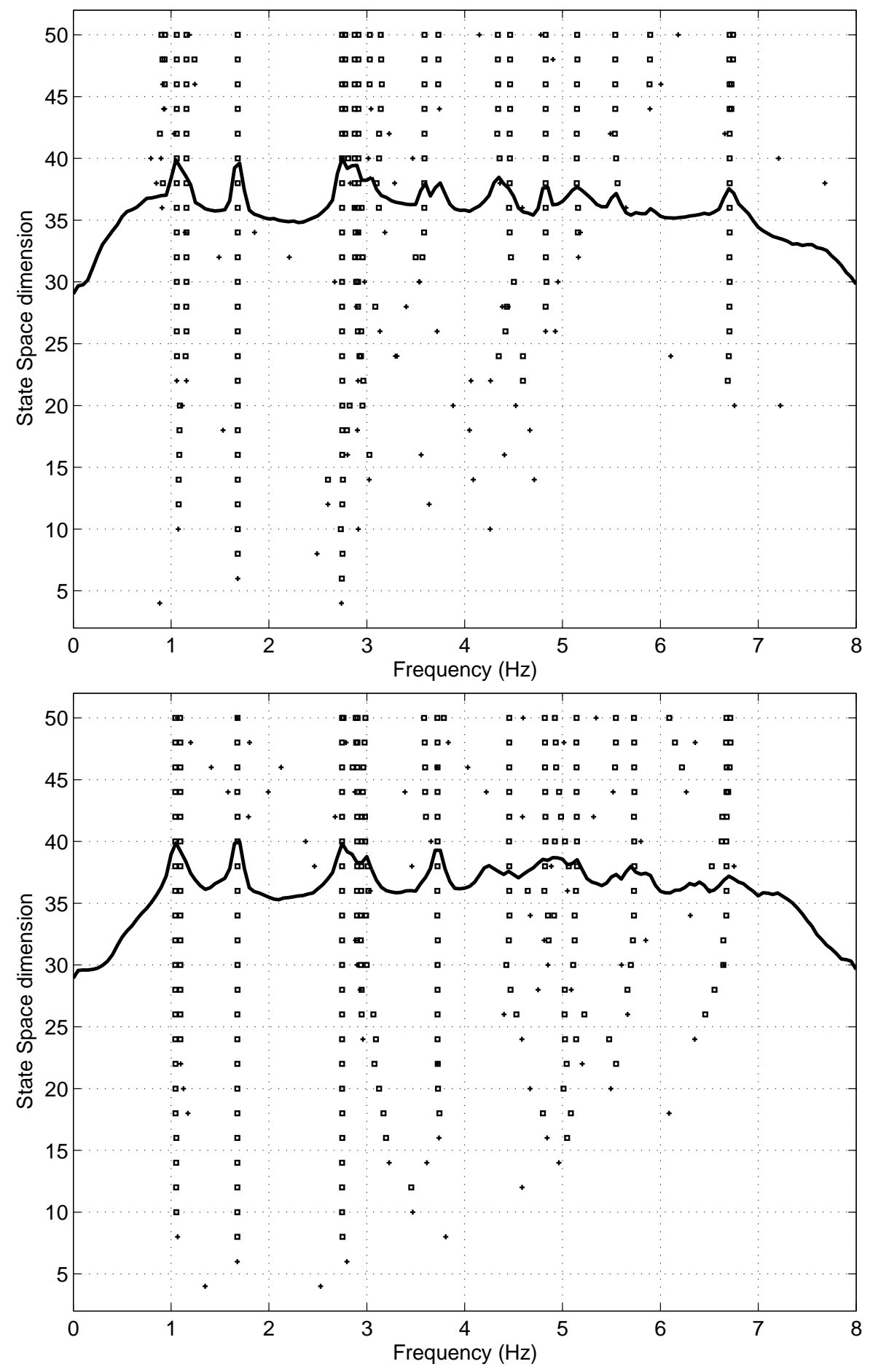

Figure 6: Stabilization diagram for set-up (T1,T2,B1) and (T1,T2,B2). The symbols " $\square$ " and "+" denote stable and unstable modes, respectively. The continuous line represents the power spectral density of the output. 


\subsubsection{Modal parameters}

This section presents the mathematical relationships between modal parameters and the estimated state space model parameters. The natural frequencies and modal damping ratios can be retrieved from the eigenvalues of A (assuming system is classically damped), and the mode shapes can be evaluated using the corresponding eigenvectors and the output matrix $\mathrm{C}$.

The eigenvalues of A are calculated as the solution of:

$$
A \psi=\lambda \psi
$$

The solution of Equation (17) consists of $n$ eigenvalues $\lambda$ and $n$ eigenvectors $\psi$ $\left\{\lambda_{1}, \psi_{1}\right\},\left\{\lambda_{2}, \psi_{2}\right\}, \ldots,\left\{\lambda_{n}, \psi_{n}\right\}$. Theoretically, these eigenvalues are complex conjugate pairs with a positive imaginary part, and consequently, the eigenvectors are complex conjugate too.

Noting that each complex eigenvalue pair of A represents one physical vibration mode, the natural frequency $\omega_{i}$ and the damping ration $\zeta_{i}$ are given by

$$
\begin{gathered}
\omega_{i}=\frac{\sqrt{\ln \lambda_{2 i-1} \ln \lambda_{2 i}}}{\Delta t}=\frac{\left|\ln \lambda_{2 i}\right|}{\Delta t} \quad i=1,2, \ldots, n / 2 \\
\zeta_{i}=-\frac{\ln \lambda_{2 i-1} \ln \lambda_{2 i}}{2 \omega_{i} \Delta_{t}}=\frac{-\operatorname{Real}\left[\ln \lambda_{2 i}\right]}{\omega_{i} \Delta_{t}} \quad i=1,2, \ldots, n / 2
\end{gathered}
$$

where $\lambda_{2 i}=\lambda_{2 i-1}^{*}\left((\bullet)^{*}\right.$ indicates complex conjugate). The $i t h$ complex mode shape $\phi_{i}$ sampled at sensor locations can be also evaluated using the following expression:

$$
\phi_{i}=C \psi_{2 i} \quad i=1,2, \ldots, n / 2
$$

where $\psi_{2 i}$ is the eigenvector of A corresponding to the eigenvalue $\lambda_{2 i}$.

\subsubsection{Stabilization diagrams}

The most popular approach for differentiating system modes from spurious noise modes is working with stabilization diagrams (Figure 6). A stabilization diagram is simply a plot of various model orders versus the frequencies identified at each of these orders. The motivation is that a system mode should show up with consistent frequency, damping and mode shape at various model orders, whereas the spurious ones could be expected to show a somewhat more erratic behavior.

The actual implementation of this strategy can be executed by initially choosing a sufficiently high order to be used in the identification problem, and then constructing smaller order models by gradually reducing the number of singular values retained. This procedure yields a set of modal parameters for each consecutive order. Parameters that belong to two consecutive model orders are then compared according to some preset criteria such as: 


$$
\begin{gathered}
\frac{\omega_{i}-\omega_{i+1}}{\omega_{i}}<\varepsilon_{\omega} \\
\frac{\xi_{i}-\xi_{i+1}}{\xi_{i}}<\varepsilon_{\xi} \\
1-M A C\left(\phi_{i}, \phi_{i+1}\right)<\varepsilon_{M A C}
\end{gathered}
$$

Modal assurance criterion of two vectors $u, v$ (denoted as $M A C(u, v)$ ) is a scalar between 0 and 1 and shows the degree to which the two vectors are correlated. If $M A C(u, v)=1$ implies perfect correlation of the two vectors (one vector is proportional to the other), while a value close to zero indicates no correlation (orthogonal vectors). $M A C(u, v)$ is computed as:

$$
M A C(u, v)=\frac{\left|u^{H} v\right|^{2}}{\left(u^{H} u\right)\left(v^{H} v\right)}
$$

where $(\bullet)^{H}$ indicates complex conjugate transpose. $\varepsilon_{\omega}, \varepsilon_{\xi}$ and $\varepsilon_{M A C}$ are some user specified tolerance limits for labeling a modal parameter as "stable". This procedure is repeated for all available sets of modal parameters identified at each order in a sequential manner, and finally, the resulting stable frequencies are plotted against their corresponding model order.

\subsubsection{Point estimates results}

Figure 6 shows the stabilization diagram for both configuration with $\varepsilon_{\omega}=2 \%$. System order and stable poles can be found is this diagram. For an order $n=30$ for the state space model we observe approximatley 10 stable poles in the frequency range of $0-8 \mathrm{~Hz}$. Frecuencies and damping ratios for $n=30$ are included in Table 1 and Table 2 labelled as $f_{\text {modal }}$ and $\xi_{\text {modal }}$.

Figure 7 represents some numerical and experimental mode shapes. MAC values for all the identified modes are included in Table 3 and Table 4. First, in Table 3, MAC values for identified modes from configuration (T1,T2,B1) and (T1,T2,B2) are calculated. It is clear that frequencies marked in gray in the table correspond to the same mode in both configurations. The correspondences between the rest of the modes are not so obvious.

Finally, Table 4 shows MAC values for identified and numerical modes. Only tree modes from numerical model are considered and the MAC show an agreement with those modes with high MAC in Table 3 (gray marks).

\subsection{Parameter estimation using confidence intervals}

A confidence interval gives an estimated range of values which is likely to include an estimated parameter with a fixed probability ( $95 \%$ for instance). Thus, confidence 


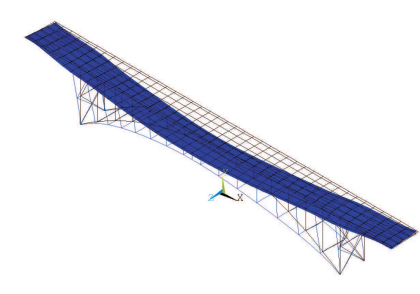

mode 1

$f=1.0564 \mathrm{~Hz}$
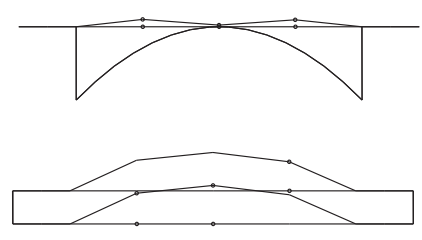

Mode $=1$

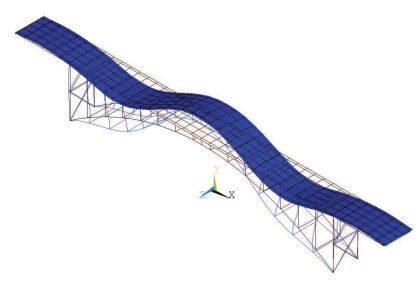

mode 2

$f=1.5625 \mathrm{~Hz}$
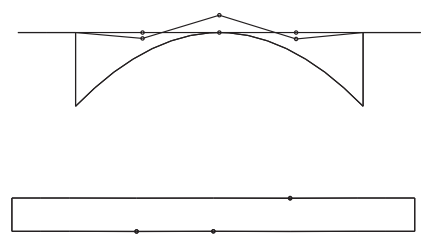

Mode $=4$

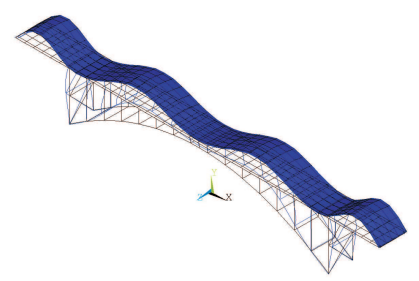

mode 3

$f=2.7610 \mathrm{~Hz}$
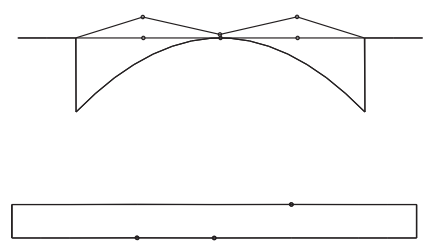

Mode $=5$

Figure 7: Mode shapes from numerical modal analysis and experimental modes for set-up $(\mathrm{T} 1, \mathrm{~T} 2, \mathrm{~B} 1)$.

\begin{tabular}{c|ccc|ccc}
\hline mode & $f_{\text {modal }}$ & $f_{\text {mean }}$ & $f_{c v}$ & $\xi_{\text {modal }}$ & $\xi_{\text {mean }}$ & $\xi_{c v}$ \\
& $\mathrm{~Hz}$ & $\mathrm{~Hz}$ & & & & \\
\hline 1 & 1.0577 & 1.0578 & 0.0011 & 0.0064 & 0.0063 & 0.1728 \\
2 & 1.1583 & 1.1584 & 0.0025 & 0.0213 & 0.0208 & 0.1153 \\
3 & 1.2356 & 1.4872 & 0.0756 & 0.6742 & 0.5017 & 0.3371 \\
4 & 1.6818 & 1.6872 & 0.0158 & 0.0020 & 0.0426 & 3.2860 \\
5 & 2.7487 & 2.7489 & 0.0003 & 0.0018 & 0.0019 & 0.2254 \\
6 & 2.9041 & 2.9009 & 0.0022 & 0.0199 & 0.0198 & 0.2302 \\
7 & 2.9118 & 2.9118 & 0.0005 & 0.0015 & 0.0023 & 1.6424 \\
8 & 3.0317 & 3.0240 & 0.0072 & 0.0728 & 0.0632 & 0.0900 \\
9 & 3.5040 & 3.4932 & 0.0187 & 0.1937 & 0.0624 & 1.6092 \\
10 & 3.5165 & 3.5773 & 0.0171 & 0.0157 & 0.1408 & 0.5198 \\
11 & 4.4679 & 4.4426 & 0.0302 & 0.0079 & 0.0222 & 4.1009 \\
12 & 4.8282 & 4.8135 & 0.0147 & 0.0048 & 0.0046 & 0.1705 \\
13 & 5.1023 & 5.0787 & 0.0112 & 0.0346 & 0.0375 & 2.0534 \\
14 & 6.7056 & 6.5939 & 0.0582 & 0.0035 & 0.0335 & 4.2221 \\
\hline
\end{tabular}

Table 1: Resulting modal parameters for configuration (T1,T2,B1)

intervals are used to indicate the reliability of an estimate instead of estimating the parameter by a single value. 


\begin{tabular}{c|ccc|ccc}
\hline mode & $f_{\text {modal }}$ & $f_{\text {mean }}$ & $f_{c v}$ & $\xi_{\text {modal }}$ & $\xi_{\text {mean }}$ & $\xi_{c v}$ \\
& $\mathrm{~Hz}$ & $\mathrm{~Hz}$ & & & & \\
\hline 1 & 1.0426 & 0.1659 & 0.0002 & 0.0060 & 0.0058 & 0.1876 \\
2 & 1.0980 & 0.1748 & 0.0005 & 0.0291 & 0.0274 & 0.1203 \\
3 & 1.1374 & 0.1850 & 0.0023 & 0.2337 & 0.2315 & 0.1123 \\
4 & 1.6777 & 0.2663 & 0.0046 & 0.0024 & 0.0048 & 4.7356 \\
5 & 2.7473 & 0.4339 & 0.0077 & 0.0027 & 0.0199 & 6.1503 \\
6 & 2.9105 & 0.4622 & 0.0015 & 0.0108 & 0.0106 & 0.2179 \\
7 & 2.9476 & 0.4688 & 0.0005 & 0.0191 & 0.0175 & 0.1449 \\
8 & 3.7292 & 0.5897 & 0.0058 & 0.0034 & 0.0038 & 0.6932 \\
9 & 4.4673 & 0.7043 & 0.0065 & 0.0338 & 0.0408 & 1.6424 \\
10 & 4.8765 & 0.7655 & 0.0042 & 0.0147 & 0.0566 & 1.3555 \\
11 & 4.9704 & 0.7959 & 0.0033 & 0.2243 & 0.0531 & 1.3405 \\
12 & 5.1067 & 0.8275 & 0.0051 & 0.0151 & 0.0655 & 1.2748 \\
13 & 5.7400 & 0.9118 & 0.0012 & 0.0270 & 0.0352 & 1.4906 \\
14 & 6.6773 & 1.0479 & 0.0060 & 0.0160 & 0.0584 & 2.1200 \\
\hline
\end{tabular}

Table 2: Resulting modal parameters for configuration (T1,T2,B2)

\begin{tabular}{c|ccccccc} 
MAC & 1.0426 & 1.0980 & 1.1374 & 1.6777 & 2.7473 & 2.9105 & 2.9476 \\
\hline 1.0577 & 0.9851 & 0.0052 & 0.0128 & 0.0005 & 0.0196 & 0.0271 & 0.1389 \\
1.1583 & 0.0000 & 0.6845 & 0.7115 & 0.0449 & 0.8101 & 0.1509 & 0.4025 \\
1.2356 & 0.0001 & 0.0805 & 0.4385 & 0.6233 & 0.1736 & 0.1918 & 0.0006 \\
1.6818 & 0.0001 & 0.0094 & 0.2784 & 0.9998 & 0.0423 & 0.6370 & 0.1496 \\
2.7487 & 0.0510 & 0.7640 & 0.8115 & 0.0387 & 0.9885 & 0.1512 & 0.3006 \\
2.9041 & 0.0166 & 0.0908 & 0.0282 & 0.4787 & 0.1332 & 0.8708 & 0.6244 \\
2.9118 & 0.0000 & 0.0852 & 0.0194 & 0.7746 & 0.0506 & 0.8693 & 0.3321 \\
\hline
\end{tabular}

Table 3: Resulting MAC for the seven first modes (rows: modes for configuration (T1,T2,B1); columns: modes for configuration (T1,T2,B2)

\subsubsection{Bootstrap procedure for state space models}

In stationary stochastic processes, the state-space formulation and the Kalman filter have yielded a modeling and estimation methodology that is much less cumbersome than the more traditional regression-based approach. In this study, the bootstrap is proposed as a method for assessing the precision of estimates of the parameters of linear state-space models. This approach employs the nonparametric Monte Carlo bootstrap [5] and is applied to subspace parameter estimation algorithm.

The stochastic system (3) can be converted into a so-called forward innovation model by applying a Kalman filter (Equation (9)): 


\begin{tabular}{c|ccc|c|ccc}
$(\mathrm{T} 1, \mathrm{~T} 2, \mathrm{~B} 1)$ & 1.0560 & 1.5630 & 2.7610 & $(\mathrm{~T} 1, \mathrm{~T} 2, \mathrm{~B} 2)$ & 1.0560 & 1.5630 & 2.7610 \\
\hline 1.0577 & 0.9772 & 0.0016 & 0.0199 & 1.0426 & 0.9436 & 0.0001 & 0.0443 \\
1.1583 & 0.0205 & 0.1509 & 0.9147 & 1.0980 & 0.0366 & 0.0089 & 0.7832 \\
1.2356 & 0.1568 & 0.0161 & 0.0542 & 1.1374 & 0.0143 & 0.0909 & 0.2692 \\
1.6818 & 0.0016 & 0.9962 & 0.1178 & 1.6777 & 0.0009 & 0.9991 & 0.0404 \\
2.7487 & 0.0003 & 0.1333 & 0.9974 & 2.7473 & 0.0010 & 0.0393 & 0.9944 \\
2.9041 & 0.0461 & 0.0198 & 0.5208 & 2.9105 & 0.0611 & 0.6174 & 0.1475 \\
2.9118 & 0.0091 & 0.7932 & 0.0000 & 2.9476 & 0.2083 & 0.1107 & 0.2457 \\
\hline
\end{tabular}

Table 4: Resulting MAC for numerical and experimental modes (rows: modes for configurations (T1,T2,B1) and (T1,T2,B2); columns: modes for numerical modes.

$$
\begin{aligned}
x_{k+1} & =A x_{k}+K e_{k} \\
y_{k} & =C x_{k}+e_{k}
\end{aligned}
$$

Here $K$ is the Kalman gain and $e_{k}$ are the innovations.

$$
\begin{gathered}
e_{k}=y_{k}-C x_{k} \\
E\left[e_{k} e_{k}^{T}\right]=\Sigma
\end{gathered}
$$

We work with the standardized innovations in a re-sampling procedure:

$$
\epsilon_{k}=\Sigma^{-1 / 2} e_{k}
$$

so we are guaranteed these innovations have, at least, the same first two moments. In Equation (28) $\Sigma^{1 / 2}$ denotes the unique square root matrix of $\Sigma$ defined by $\Sigma^{1 / 2} \Sigma^{1 / 2}=$ $\Sigma$.

The Monte Carlo bootstrap procedure for state-space models is defined by a five step algorithm. We assume that the model estimation has been completed and we have an estimation for $A$ and $C$, and for the initial conditions $x_{1}^{0}$ and $P_{1}^{0}$ :

1. Estimate the innovation sequence $e_{k}$ and the states sequence $x_{k}$ from Equation (26) and (25).

2. Construct the standardized innovations by setting

$$
\epsilon_{k}=\Sigma^{-1 / 2} e_{k}
$$

where $\Sigma^{-1 / 2}$ is the inverse of the unique square-root matrix of $\Sigma$.

3. Sample with replacement from $\epsilon_{k}$ to obtain a bootstrap sample of standardized innovations, $\epsilon_{k}^{*}$ (this is the Monte Carlo step). 
4. Construct a bootstrap data set $y_{k}^{*}$ using $\epsilon_{k}^{*}$ in place of $\epsilon_{k}$ in Equation (25). The state variables, $x_{k}$, and the initial conditions of the Kalman filter remain fixed at their given values $x_{1}^{0}$ and $P_{1}^{0}$.

5. Repeat steps (3) and (4) a large number, N, of times, obtaining a set of parameter replications, $\left\{\hat{\theta}^{* i} ; 1 \leq i \leq N\right\}$. Estimate the distribution of the parameters from the distribution of the $\hat{\theta}^{* i}$.

\subsubsection{Interval estimates results}

Table 1 and Table 2 show $f_{\text {mean }}$ and $f_{c v}$ for the mean value and for the coefficient of variation of frequency calculated using Bootstrap Montecarlo procedure. The same results are obtained for damping ratio $\left(\xi_{\text {mean }}, \xi_{c v}\right)$.

A plot similar to stabilization diagram is included in Figure 8 where the frequencies identified in each bootstrap replication are plotted. These graphics differentiate system modes from spurious noise modes for a given order system.

\section{Conclusions}

The firs aim of this paper was to provide an assessment of the stochastic subspace methods for ambient vibration analysis. The motivation was to objectively determine the practical beneficits of subspace methods and find out the potential difficulties associated with output only modal identification. To this end, investigations were performed in a steel arch bridge.

The second aim was to develop a method for assessing the precision of estimates of the parameters of state-space models (interval estimate). This approach employs the nonparametric bootstrap procedure. The results of interval estimate revealed that the damping estimates are usually identified with much less accuracy than the frequencies.

One important problem in real life structures is differentiating true structural modes from spurious noise modes. The stabilizations diagrams proved to be useful tools for viasually choosing the number of significant modes. Using bootstrap results, a plot similar to stabilization diagram was presented. These graphics differentiate system modes from spurious noise modes for a given order system.

Additionally, using Modal Assurance Criterion, the obtained experimental modes has been compared with those evaluated from a finite element analysis. A quite good agreement between numerical and experimental results is observed.

\section{Acknowledgements}

This research was supported by Spanish Ministerio de Fomento. The authors are indebted especially to the engineer coordinating the project, Mrs. Pilar Crespo, whose support and advice are gratefully acknowledged. 

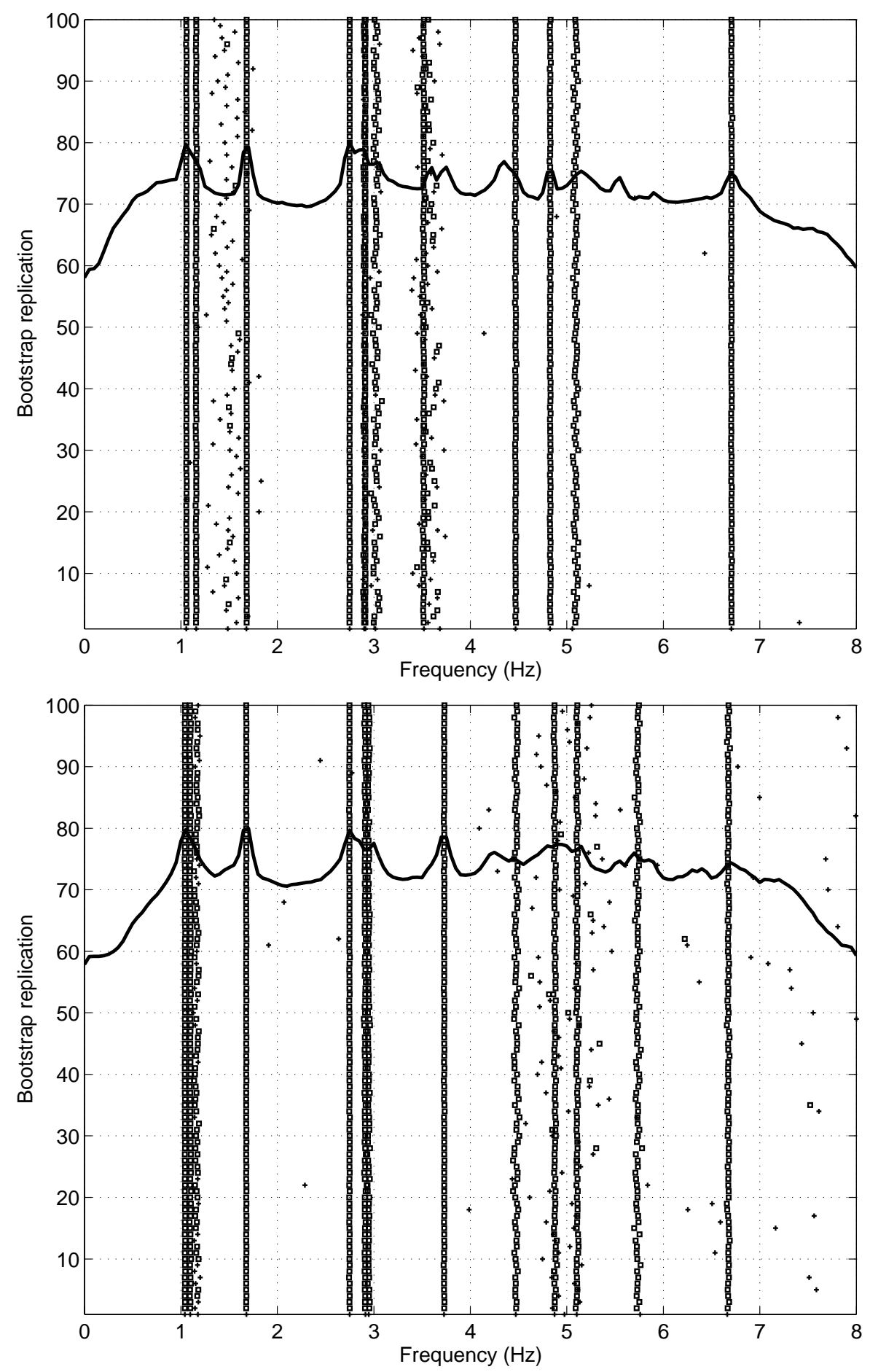

Figure 8: Stabilization diagram using Bootstrap results for set-up (T1,T2,B1) and (T1,T2,B2). The symbols " $\square$ " and "+" denote stable and unstable modes, respectively. The continuous line represents the power spectral density of the output. 


\section{References}

[1] L. Ljung, "System Identification. Theory for the users". 2nd Ed., PTR PrenticeHall, Upper Saddle River, N.J. 1999.

[2] P. Van Overschee and B. De Moor, "Subspace Identification for Linear Systems. Theory - Implementation - Applications". Kluwer Academic Publishers. 1996.

[3] R.H. Shumway and D.S. Stoffer, "Time series analysis and its applications". Springer. 2006.

[4] R. Brincker, L. Zhang and P. Andersen, "Modal identification from ambient responses using frequency domain decomposition". Proceedings of IMAC 18, the International Modal Analysis Conference, San Antonio, TX. 2000.

[5] D.S. Stoffer and K.D Wall, "Bootstrapping State-Space Models: Gaussian Maximum Likelihood Estimation and the Kalman filter". Journal of the American Statistical Association. 1991. Vol. 86, No. 416.

[6] P. Galvín, J. Domínguez, "Dynamic analysis of a cable-stayed deck steel arch bridge". Journal of Constructional Steel Research, 63. 1024-1035. 2007.

[7] B. Alicioglu and M. Lus, "Ambient vibration analysis with subspace methods and automated mode selection: case studies". Journal of Structural Engineering. ASCE. June 2008.

[8] X. He et all, "System identification of alfred Zampa Memorial Bridge using dynamic field test data". Journal of Structural Engineering. ASCE. January 2009.

[9] . T. Katayama, "Subspace Methods for System Identification". Springer-Verlag London. 2005.

[10] J. Li and Y. Zhang, "Prediction error method-based second-order structural identification algorithm in stochastic state space formulation". Earthquake Engineering and Structural Dynamics. 1991. 2006. 35:761-779. 